\title{
Restructuring a New Cognitive Frame Work of the Neuropathogenesis of Atopic Dermatitis and Its Implications
}

\author{
Chan Kam Tim Michael* \\ Specialist in Dermatology, Hong Kong Academy of Medicine, China
}

Received: June 18, 2018; Published: June 26, 2018

*Corresponding author: Chan Kam Tim Michael, Specialist in Dermatology, Hong Kong SAR, China. 2128, Pioneer Centre, 750, Nathan Road, Mongkok, Kowloon, Hong Kong, Hong Kong SAR, China

\begin{abstract}
Abbreviations: AD: Chronic Atopic Dermatitis; MRSA: Methicillin Resistant Staphylococcus Aureus;PNS: Peripheral Nervous System; CNS: Central Nervous System; TRP: Transient Receptor Potential; SP: Substance P; NO: Nitric Oxide;DRG: Dorsal Root Ganglion; CGRP: Calcitonin Gene Related Peptide; H1: Histamine 1; NNP: Non-Histaminergic Neuronal Pathway; PAR 2: Proteases Activated Protein; TSLP: Thymic Stromal Lymphopoietin;Ca++:CalciumCation Influx; Na+: Sodium;GRPR: Gastrin Releasing Peptide Receptor; NGF: Nerve Growth Factors; NA: Noradrenaline; IFN-Y: Interferon-Y; Nppb: Natriuretic Polypeptide b; f-MRI: Functional Magnetic Resonance Imaging; AI: Artificial Intelligence; T-DCS: Transcranial Direct Current Stimulation; R - TMS: Repetitive Transcranial Magnetic Stimulation; EMG:Electromyography
\end{abstract}

\section{Short Communication}

Chronic Atopic Dermatitis (AD) is inadequately managed in the primary health care and hospital settings [1,2]. Steroid phobia, emergence of methicillin resistant Staphylococcus aureus (MRSA), poor compliance to emollients therapy, inaccessibility to costly biologics and lack of effective preventive measures are all real problems to service providers taking care of children and adults suffering from this chronic distressing illness. Behavior disturbances, fear, anxiety, social isolation, stress and depression are negative emotional factors well reported associated with intractable pruritus of chronic $\mathrm{AD}$ [3]. The current treatments protocol focusing on the skin with its epidermal barrier and immunological aberrancy and hypersensitivity may not be sufficient to face up the present challenges. An escalating prevalence of chronic AD had been reported in most populated cities.In view of the aforesaid, the advent of non-invasive functional-MRI and related neuro-cognitive investigations, a new cognitive-mind-movement behaviour and skin inflammation neuro pathogenetic framework is suggested. This top down; cognitive - mind patient centered approach placed the objective of management of individual chronic AD sufferers to a more non-pharmacological, multi-disciplinary team approach procuring modern technologies with an aim to reconstruct the mind and cognition of patients, parents and the health care providers. Patients, parents, nurses, practitioners and the whole health care team are integrally educated on itch - scratch and itch - anxiety - scratch cognitive phenomenon of chronic AD. This new focus is very much different from the current disease - based approach practicing by front line practitioners, pediatricians and dermatologists treating solely the skin inflammation by immune-modulators, antibiotics and systemic antihistamines. The top down holistic approach is evidence based as researchers now have good evidences that a pruritogenic circuitry existed that linked up the central nervous system (CNS) to our outermost layer, the skin.

\section{Existence of a Pruritogenic Specific Neuron Signalling Pathway}

\section{Pruritogenic Signal Transmission in the Skin}

Starting from the epidermis: keratinocytes; morphologically and functionally; is the outermost sensors of the peripheral nervous system (PNS) [4,5]. Epidermal keratinocytes possessed the widely distributed transient receptor potential (TRP); TRPV 1 receptors readily receiving environmental noxious stimuli including, heat, chemicals, pain and itch [5]. Depolarization signals through calcium $(\mathrm{Ca}++)$ cation influx. Free nerve endings belong to the small neuro $\mathrm{C}$ fibers are posited between individual keratinocytes, sending neuronal signals through its afferent neurons joined by its counterparts of neuronal receptors of the PNS in the skin dermis. Nerve free endings of the afferent neurons possess TRPV 1 receptors which are activated to secret Substance P(SP), nitric oxide (NO) locally and in the dorsal root ganglion (DRG) neurons. SP is known to be a potent pruritogenic neurotransmitter which triggers the glutamate pathway and by calcium influx through the NMDA; calcium channel propagated the sensation of pruritus. Calcitonin Gene Related Peptide (CGRP) and tachykinin are also produced. 
SP may further provoke a cascade of local tissue inflammatory responses through a number of mediators, cytokines (IL -1, 2, 4, 13,31 , etc) and molecules which are known induce pruritus. This is referred to TRPV1 neurogenic inflammatory pathway of pruritus [6]. The discoveries of Mrgrp receptors bring insights that peripheral itch sensory inputs are not only histamine neurons mediated but non-histamine neurons mediators mediated $[7,8]$ Histamine 1 (H1) was shown to interact with TRPV 1 transmitting pruritic neuronal signals to the CNS [9]. The non-histaminergic neuronal pathway (NNP) is more complicated and not completely elucidated. NNP involved afferent neurons possessing other neuronal receptors like TRPA 1, proteases activated protein (PAR 2), Endothelial 1, Thymic stromal lymphopoietin (TSLP) and serotonin receptors [9]. MrgprA3 and MrgprC11 interact with TRPV 1 and TRP ankyrin 1 (TRPA1) in the epidermis transmit histaminergic and non-histaminergic pruritic signals to the CNS [9]. Selectivity model posits that itch and pain neurons are polymodal, with itch sensations occurring when selective subsets of itch neurons are activated alone and pain sensations dominating when itch and pain neurons are activated together. In most circumstances, intense pain suppresses sensation of pruritus. A subset of itch specific neuron exists exclusively transmit pruritic signal peripherally to the spinal cord of CNS [10]. Currently, two voltage gated depolarization signals; one involved $\mathrm{Ca}++$ cation and the other sodium $(\mathrm{Na}+)$ cation have been identified; in the transmission of pruritus.

\section{Pruritogenic Circuitry in Spinal Cord}

Pruritogenic afferent neurons then synapse with neurons in the DRG through Gastrin releasing peptide receptor (GRPR) inter-relay pruritic neuronal signals contralateral to the opposite side of the same level of DRG of the spinal cord [11]. Gastrin releasing peptide plays a key role and is a robust mediator of pruritus in CNS. An Inhibitory inter - neuronal pathway Bhlhb5+ (B5-I) inhibits the transduction of pruritus through downregulation of TRP channels with the release of a kappa opioid receptor ligand neuropeptides called dynorphin to impede pruritus as well as pain [12]. A vast array of inflammatory mediators like prostaglandins, bradykinin, SP, nerve growth factors (NGF), cytokines, insulin, serotonin, noradrenaline (NA), interferon- $\Upsilon$ (IFN- $\Upsilon$ ), histamine 1 to 4 , proteases and toll like receptors and its ligands secreted by immunological activated mast cells, T lymphocytes, keratinocytes, granulocytes and macrophages. Many other mediators that promote pruritus in the DRG are SP, cytokines, interleukins especially IL-31 and natriuretic polypeptide $b$ (Nppb) expressed in a subset of TRPV 1 neurons. Chronic pruritus promotes glial cell proliferation and increase astrocytes proliferation especially following induction of the body inflammasome in the CNS during chronic pruritus [13,14]. Reactive astrogliosis in the spinal cord glial cells occurs during chronic scratching behaviour.

\section{The CNS Pruritogenic Circuitry}

The thalamus, midbrain and the autonomic nervous system is the center of transmission of pruritus in CNS. The contralateral ascending spinothalamic tract relay histaminergic and non-histaminergic pruritogenic signals to the inner structures of the brain. Hedonic scratch activated the primary somatosensory S1 areas of the cerebral cortex give the perception of pleasantness of pruritus in the cingulate cortex which decided the movement of scratching from the motor cortex. The pain results from chronic scratchings physiologically inhibited the pruritus sensation. The midbrain, ventral tegmental area, striatum, nucleus, accumbens, caudate nucleus, ventromedial prefrontal cortex, insula and claustrum are all shown by functional magnetic resonance imaging (f-MRI) studies to be activated in this rewarding circuitry [15].

\section{Itch-Anxiety - Cycle in Amygdala}

Itch- anxiety circuitry existed in the primitive part of our inner brain, involving the hippocampus; its adjacent anatomical structures like amygdala, anterior cingulate cortex and insula cortex during itch and anxiety [9]. Subcallosal gray matter and nucleus accumbens of the brain are anatomically and physiologically activated in depression during chronic scratchings. Specific anatomical areas of the prefrontal cortex can execute inhibitory signals to amygdala to suppress fear, anxiety, stress and chronic pruritus via a top down regulation through co-ordination of different cognitive domains in the cerebral cortex [16]. Imbalance and miswiring of various brain neurotransmitters like GABAs, serotonin, noradrenaline and dopamine has been implicated in mediating chronic pruritus in the brain through this complicated multi-dimensional pruritic circuitry [17].

\section{Pharmacological and Non-Pharmacological Implica- tions}

Chronic AD is a cognitive phenomenon mediated through neuroendocrine mediators. A specific neuronal pruritic circuitry anatomically and functionally existed involved the skin, brain, cognition, mind and movement [18]. An armamentarium of modern technologies and medicines are available. Early prevention, diagnosis, patient orientated multi-disciplinary approach may be one of the most cost-effective management strategy. We advocate nurses, parents and partners participation in the care management of chronic AD. Education, counselling and explanation of the physiological nature of pruritus in the form pamphlets, videos and focus groups are recommended. Currently, in the literature the following non-pharmacological methods have been reviewed including positive motivational psychotherapy, refocusing attention strategies, virtual reality immersion, audio-visual distraction techniques, habit reversal training, arousal reduction, cognitive restructuring and utilization of artificial intelligence (AI) [19-21].

The cognitive restructuring method should be evident based, non-invasive, patient's acceptance, convenient and cost-effective. Transcranial direct current stimulation (t-DCS) and repetitive transcranial magnetic stimulation (r-TMS) anti-pruritic technologies have also been studied [22]. Looking into the future, AI with the utilization of Apps; for example; involving the readily accessible Google platform; real time detection of pruritic itch-cognitive -movement behaviour can be accurately recorded in the form of signals like electromyography(EMG) in a timely manner with life events circumstances like day time workings, resting, sleeping and emotional upsets; regardless the patients conscious or subconscious cognitive states. The results and the pattern of pruritus in relation 
to different life attributes can be systemically analyzed, studied and explained to the chronic AD pruritic team and the patients.

Simple, evidence based, non-toxic topical pharmacological therapies like moisturizers applications can be enhanced.In sum; chronic $\mathrm{AD}$, starting from scratch; in addition to genetic mutations of epidermal scaffolding protein like filaggrin and involucrum; barrier defects, early stages of immunoglobin Ig E sensitization; is an itch - cognition - mind - movement - scratch - pain - inflammatory viscous pathophysiological events. A personalized, positive interventional psychotherapy involving restructuring the cognition of the patients and care providers should have an important management role of this distressing, difficult - to - control condition.

\section{References}

1. Holm JG, Anger T, Clausen M L, Thomsen SF (2016) Quality of life and disease severity in patients with atopic dermatitis. J Eur Acad Dermatol Venereol 30(10): 1760-1767.

2. Weidinger S, Novak N (2016) Atopic Dermatitis. Lancet 387(10023): 1109-1122.

3. Sanders KM, Akiyama T (2018) The vicious cycle of itch and anxiety. Neuroscience and Biobehavioral Reviews 87: 17-26.

4. Baumbauer KM, DeBerry JJ, Adelman PC, Miller RH, Hachisuka J, et al. (2015) Keratinocytes can modulate and directly initiate nociceptive responses. Elife 2: 4 .

5. Tóth BI, Oláh A, Szöllősi AG, Bíró T (2014) TRP channels in the skin. Br J Pharmacol 171(10): 2568-2581.

6. Geppetti P,Nassini R, Materazzi S, Benemei S (2008) The concept of neurogenic inflammation. BJU Int. 101(3): 2-6.

7. Liu Q, Tang Z, Surdenikova L, Kim S, Patel KN, et al. (2009) Sensory neuron-specific GPCR Mrgprs are itch receptors mediating chloroquineinduced pruritus. Cell 139(7): 1353-1365.

8. Qu L, Fan N, Ma C, Wang T, Han L, et al. (2014) Enhanced excitability of MRGPRA3 - and MRGPRD-positive nociceptors in a model of inflammatory itch and pain. Brain 137(4): 1039-1050.

9. DM Bausita, Sarah R Wilson, Mark A Hoon (2014) Why we scratch an itch: the molecules, cells and circuits of itch. Nature Neuroscience. Volume 17(2): 175-182.

This work is licensed under Creative Commons Attribution 4.0 License

Submission Link: https://biomedres.us/submit-manuscript.php
10. Sanders KM, Nattkemper LA, Yosipovitch G (2016) Advances in understanding itching and scratching: a new era of targeted treatments. F1000Res 5: 1-7.

11. Sun YG, Chen ZF (2007) A gastrin-releasing peptide receptor mediates the itch sensation in the spinal cord. Nature 448(7154): 700-703.

12. Kardon AP, Polgár E, Hachisuka J, Snyder LM, Cameron D, et al. (2014) Dynorphin acts as a neuromodulator to inhibit itch in the dorsal horn of the spinal cord. Neuron 82(3): 573-586.

13. ShiratoriHayashi M, Koga K, TozakiSaitoh H, Kohro Y, Toyonaga H, et al. (2015) STAT3-dependent reactive astrogliosis in the spinal dorsal horn underlies chronic itch. Nat Med 21(8): 927-931.

14. Zhang Y, Dun SL, Chen YH, Luo JJ, Cowan A, et al. (2015) Scratching activates microglia in the mouse spinal cord. J Neurosci Res 93(3): 466474.

15. Mochizuki H, Schut C, Nattkemper LA, Yosipovitch G (2017) Brain mechanism of itch in atopic dermatitis and its possible alteration through non-invasive treatments. Allergology International 66(1): 1421.

16. Sanders KM, Akiyama T (2018) The vicious cycle of itch and anxiety. Neuroscience and biobehavioral Reviews 87: 17-26.

17. Chen L, Wang W, Tan T, Han H, Dong Z (2016) GABA(A) receptors in the central nucleus of the amygdala are involved in pain- and itch-related responses. J Pain 17(2): 181-189.

18. KTM Chan (2018) A concise review and update of the anatomical circuitry of itch. J Anat Physiol Stud 1(1).

19. Steinhoff Martin, Ferda Cevikbas, Iwei Yeh, Kim Chong, Jörg Buddenkotte, et al. (2012) Evaulation and management of a patient with chronic pruritus. J Allergy Clin Immunol 130(4): 1015-1016.

20. Leibovici V, Florella Magora, Sarale Cohen, Arieh Ingber (2009) Effects of virtual reality immersion and audiovisual distraction techniques for patients with pruritus. Pain Res manage 14(4): 283-286.

21. Schut C, Mollanazar NK, Kupfer J, Gieler U, Yosipovitch G (2016) Psychological interventions in the treatment of chronic itch. Acta Derm Venereol 96(2): 157-161.

22. Nakagawa K, Mochizuki H, Koyama S, Tanaka S, Sadato N, et al. (2016) A transcranial direct current stimulation over the sensorimotor cortex modulates the itch sensation induced by histamine. Clin Neurophysiol 127(1): 827-832.

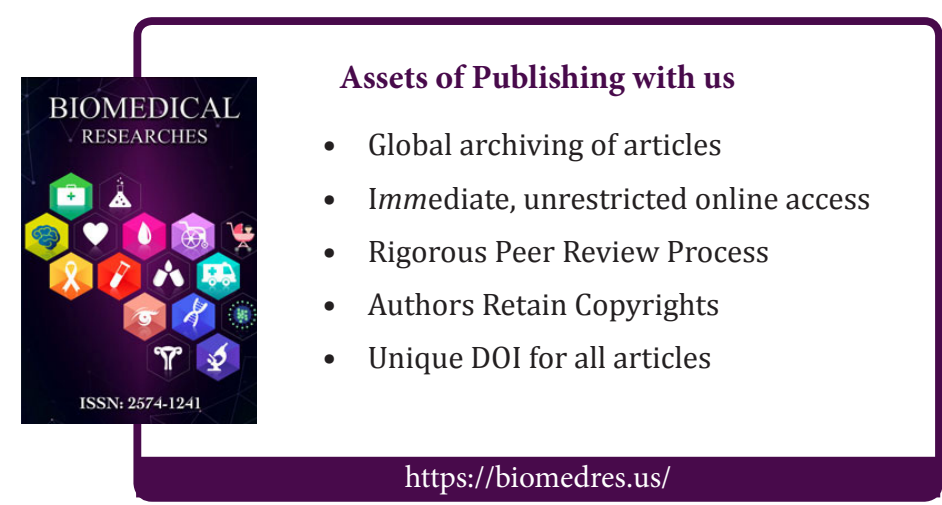

